\title{
EL SÍNDROME DEL "QUEMADO" POR ESTRÉS LABORAL ASISTENCIAL EN GRUPOS DE DOCENTES UNIVERSITARIOS
}

\author{
Carlos Ramón Ponce Díaz; Mario Santiago Bulnes Bedón; Jaime Ramiro Aliaga Tovar; \\ María Clotilde Atalaya Pisco; Rosa Elena Huertas Rosales
}

\begin{abstract}
RESUMEN
Objetvos: Conocer la posible presencia del síndrome del "quemado" por estrés laboral asistencial en grupos de docentes de una Universidad Nacional, pertenecientes a diversas áreas de especialidad. Describir posibles diferencias significativas en el síndrome del "quemado" por estrés laboral asistencial, según áreas de especialidad y en relación a las variables Sexo, Estado Civil, Práctica de Deporte, Problemas de salud y Condición docente. Diseño: Es descriptivo, comparativo y correlacional, de carácter analítico, realizado en profesores universitarios distribuidos en cinco áreas de especialidad en el cual se trato de detectar la presencia e implicancias del síndrome del "Burnout".
\end{abstract}

Material y métodos: En el presente estudio se ha trabajado con una muestra de 274 docentes de la Universidad Nacional Mayor de San Marcos, pertenecientes a cinco áreas de especialidad: Ciencias de la Salud; Básica; Ingenierías; Letras-Educación-Derecho y Administración-Economía-Contabilidad, respectivamente y que ha sido seleccionada a través de un muestreo no-probabilístico de tipo intencional o intencionado. El instrumento utilizado ha sido el MBI-Inventario "Burnout" de Cristina Maslach y Susan Jackson, el cual comprende una escala tridimensional y a partir de ella se han definido las siguientes escalas o variables del sujeto "quemado": Cansancio emocional (CE), despersonalización (DP) y realización personal (RP).

El análisis estadístico se llevó a cabo en tres etapas: Análisis descriptivo y exploratorio para describir de manera detallada la distribución de la muestra examinada, análisis psicométrico del MBI-Inventario "Burnout" de Maslach y Jackson, y análisis inferencial para la contrastación de hipótesis.

Resultados: En el MBI-Inventario de "Burnout" de Maslach y Jackson, los resultados no se aproximan a la distribución normal, por lo que se ha trabajado la investigación con estadísticos no paramétricos sobre la base de rangos. Los resultados corroboran que los ítemes del inventario son consistentes entre sí. El análisis de la confiabilidad por consistencia interna nos indica coeficientes Alfa razonables en las tres escalas o variables del inventario de "Burnout", los que nos permite inferir la existencia de puntajes confiables. El análisis factorial exploratorio nos revela que existe un solo factor bipolar que tiene una varianza que explica el $54.5 \%$ de la varianza total la cual corrobora la presencia del síndrome del "Burnout". Conclusiones: Los docentes de la Universidad Nacional Mayor de San Marcos presentan evidencias del síndrome del "quemado" por estrés laboral asistencial. El análisis cualitativo permite apreciar diferencias en la presencia del "Burnout" según áreas de especialidad. No existen diferencias en las etapas del "Burnout" en relación a la variables Sexo, Estado Civil 
y Práctica de Deporte. Existen diferencias significativas en las etapas del "Burnout" en relación a las variables Problemas de Salud y Condición Docente. A mayor tiempo de servicios y a mayor tiempo en la profesión, se detecta: menor cansancio emocional, menor despersonalización y mayor realización personal, y viceversa. Los docentes que tienen problemas de salud infieren mayor cansancio emocional y mayor despersonalización.

Palabras claves: "Burnout", estrés, docente universitario, inventario, análisis factorial.

\begin{abstract}
Objective: To acknowledge the possible presence of the "Burnout" syndrome caused by existential work stress in university professors group of a state university who belong to
\end{abstract} different career area.

To find possible significant differences in the "Burnout" syndrome caused by existential work stress in different career area and according to the variable: sex, marital status, sport practice, health and teaching condition.

Design: It is descriptive, comparative, correlational and analytic preformed on university proffesors distributed in 5 carrer area, in which an effort was mad to find the presence and implication of the "Burnout" syndrome.

Instruments and methods: The present study has used a sample of 274 professors of Universidad Nacional Mayor de San Marcos (UNMSM), that belong to 5 different career area: humanities-education-law and business administration-economics-accounting, and was selected through a non-probabilistic sample of an intentional type. The tool used by the present study has been the MBI-"Buraout" Inventory created by Cristina Maslach and Susan Jackson, which uses a tri-dimensional scale that defined the following scales or variables of the "Burnout" subjectemotional tiredness, de-personalization and personal fulfillment.

The statistic analysis was done in 3 stages: descriptive and exploratory analysis to describe in detail the sample distribution; psychometric analysis of the MBI-"Burnout" Inventory by Maslach and Jackson; and inferential analysis for hypothesis contract. Results: In the MBI"Burnout" Inventory by Maslach and Jackson the results do not approximate to the standard distribution, for which the study has worked with non-parametric statistics on a range base.

The results prove that the items from the inventory are consistent among them. The probability and internal consistency analysis shows us alfa reasonable coefficients in the 3 scales or variables of the "Burnout" Inventory, which allows us to infer the existence of trust worthy scores. The factorial exploratory analysis reveals us that there is only 1 bipolar factor that has a variance that explains $54.5 \%$ of the total variance, which proves the presence of the "Burnout" syndrome.

Conclusions: The UNMSM professors show evidence of the "Burnout" syndrome caused by existential work stress. The qualitative analysis allows us to see the differences in presence of the "Burnout" syndrome, according to each carrer area. There are certain differences in the "Burnout" stages in relation to the variable: sex, marital status and sports variable: health problems and teaching conditions. If there is a longer service time and job position we find less emotional tiredness, less de-personalization and more personal fulfillment. Professors with health problems show greater emotional tiredness and depersonalization.

Keywords: "Burnout", stress, university professors, inventory, factorial analysis. 


\section{INTRODUCCIÓN}

En las últimas décadas, se ha desarrollado un gran interés por el estudio del estrés y los factores psicosociales relacionados con el trabajo y la repercusión de éstos sobre la salud de los trabajadores. Sobre el punto, debemos señalar que el interés por la productividad y la eficiencia, no siempre han sido acompañados de condiciones laborales dignas y adecuadas, recursos suficientes para llevarlo a cabo y un diseño de tareas y puestos que tengan en cuenta las características de las personas, sus necesidades, habilidades e intereses. La falta de atención a estos aspectos pueden generar consecuencias sobre las personas (estrés, insatisfacción), o sobre la empresa (ausentismo, conflictividad, disminución de la calidad del trabajo y la productividad, etc.).

Dentro de las enfermedades asociadas al estrés laboral, el síndrome de "Burnout", también llamado "síndrome de estar quemado", "síndrome de la quemazón", "síndrome del estrés laboral asistencial" o "síndrome del desgaste profesional", es cada vez más conocido y lamentablemente más extendido, y suele afectar principalmente a personas cuyas tareas laborales tienen un componente central de ayuda a los demás y de gran intervención social como los médicos, enfermeros, odontólogos y demás profesionales de la salud, así como a docentes, trabajadores sociales, deportistas, policías, entre otros.

El síndrome del "Burnout", puede considerarse como el mal del fin de siglo, al punto que se calcula que alrededor de dos tercios de todas las enfermedades están relacionadas con el estrés laboral, y aunque no se trata de una relación de causa-efecto, el estrés laboral es un factor importante que interacciona con otras variables biológicas, psicológicas y sociales, dando lugar a numerosas enfermedades físicas y mentales. Inclusive, existen referencias que nos informan que el estrés laboral es un problema grave que está vinculado con el origen del $50 \%$ de las bajas laborales en la Unión Europea.

El síndrome del "Burnout", mal invisible que afecta y repercute en la calidad de vida, es considerado como la fase avanzada del estrés profesional y se produce cuando se desequilibran las expectativas en el ámbito profesional y la realidad del trabajo diario, produciéndose desmotivación, desinterés, malestar interno e insatisfacción laboral en el sujeto que lo padece, el cual infiere "tensión" al interactuar y al tratar reiteradamente con otras personas.

A diferencia de un estrés puro, el "Burnout" siempre está asociado a sentimientos de desvalorización y fracaso. El síndrome del "Burnout" se manifiesta bajo unos síntomas específicos, siendo los más habituales:

A. Psicosomáticos: Cansancio, fatiga crónica, frecuentes dolores de cabeza, malestar general, problemas de sueño, contracturas y algias óseo musculares, ulceras y otros desórdenes gastrointestinales, perdida de peso, taquicardia, hipertensi6n, etc.

B. Conductuales: Mala comunicación, ausentismo laboral, abuso de drogas (café, tabaco, alcohol, fármacos, etc.), incapacidad para vivir de forma relajada, superficialidad en el contacto con los demás, aumento de conductas violentas, trastornos en más o en menos del apetito y la ingesta, distanciamiento afectivo de los clientes y compañeros.

C. Emocionales: Distanciamiento afectivo como forma de protección del yo, aburrimiento y actitud cínica, impaciencia e irritabilidad, sentimiento de omnipotencia, desorientación, incapacidad de concentración, disminución de la memoria inmediata, baja tolerancia a la frustración, sentimientos depresivos, sentimientos de vació, agotamiento, fracaso, impotencia, baja autoestima y pobre realizaci6n personal. 
D. Laborales: Detrimento en la capacidad de trabajo, detrimento en la calidad de los servicios que se presta a los clientes, aumento de interacciones hostiles, comunicaciones deficientes, frecuentes conflictos interpersonales en el ámbito del trabajo y dentro de la propia familia.

Las evidencias que afectan al individuo en el inicio de la aparición de la enfermedad, se reconocen en varias etapas y son:

1. Exceso de trabajo.

2. Sobreesfuerzo que lleva a estados de ansiedad y fatiga.

3. Desmoralización y pérdida de la ilusión.

4. Pérdida de vocación, decepción de los valores hacia los superiores.

Herbert J. Freudenberger (1974), psicoanalista alemán residente en Norteamérica y que trabajaba en una clínica neoyorquina, fue el que introdujo, desde una perspectiva clínica, el termino "Burnout" en la literatura científica para hacer referencia a un conjunto de manifestaciones anómalas características de las personas que trabajan en profesiones de ayuda a los demás ("helping professions"). Definió el síndrome como "un conjunto de síntomas médico-biológicos y psicosociales inespecíficos, que se desarrollan en la actividad laboral, como resultado de una demanda excesiva de energía".

Para él, se trata de "una sensación de fracaso y una experiencia agotada que resulta de una sobrecarga por exigencias de energía, recursos personales o fuerza espiritual del trabajador". Como podemos apreciar, la definición que nos brinda se refiere a un estado emocional que se encuentra al final de un proceso. Para dicho autor, este proceso que acaba en el "Burnout" se debe a una elevada implicaci6n del trabajador en su profesión, la "adicción al trabajo".

En concreto, se refirió a los profesionales denominados de "ayuda" cuya actividad va dirigida a otras personas, con las que mantienen fuerte y sostenido contacto directo. Sus primeras observaciones fueron los efectos que en poco mas o menos de un año sufrían muchos de los voluntarios: Una progresiva pérdida de energía que llevaba al agotamiento, ansiedad inespecífica y, en algunos casos, cuadros de tipo depresivo, en un marco de pérdida de la motivación e interés con que abordaron su trabajo, e incluso manifestando intolerancia, resentimiento y agresividad con los pacientes.

A partir de esa fecha, el síndrome del "Burnout" ha generado infinidad de trabajos, especialmente en el ámbito organizacional. Casas \& Hilari, 2002, informa que algunos artículos realizados en 1990 citan más de 2500 referencias sobre "Burnout" entre sus primeros estudios, en 1974 y 1989. Ello se debe a la importancia que merece el tema para el mundo empresarial, donde los costes debido al "burnout" pueden suponerse elevados, a pesar de poseer escasos estudios que evalúen concienzudamente las repercusiones del síndrome en las organizaciones.

Por otra parte, se han desarrollado diversos intentos para alcanzar una delimitación te6rica del constructo (concepto, expresividad, factores implicados en su desarrollo, etc). Algunos autores explican el síndrome como consecuencia del fallo en las estrategias de afrontamiento al estrés laboral. En tal sentido, Edelwich y Brodsky,1980 sostienen que el síndrome de desgaste profesional sería: "como una pérdida progresiva del idealismo, energía y motivos vividos por la gente en las profesiones de ayuda, como resultado de las condiciones de trabajo". 
Estos mismos autores proponen cuatro fases por las que supuestamente pasaría una persona en un proceso de desgaste profesional: entusiasmo; estancamiento; frustración y apatía.

Otras propuestas, por aclarar el concepto, intentan una clasificación del síndrome en dos categorías diferenciadas: "el Burnout activo", que se demuestra por una manifestación del desgaste a través de conductas asertivas, con muestras de irritabilidad y hostilidad; y el "Burnout pasivo", que viene caracterizado por las conductas de retirada y la apatía. A estas alturas debemos tener presente, según Guerrero Barona, 1998, que el "Burnout", es un término anglosajón cuya traducción mas próxima y coloquial es "estar quemado", desgastado, exhausto y perder la ilusión por el trabajo.

En virtud a lo mencionado anteriormente, la aproximación más fructífera ha sido la realizada por Maslach y Jackson (1981), quienes desde una perspectiva psicosocial subrayan la dimensión emocional del "burnout" como forma de respuesta al estrés. En este sentido, el "burnout" es concebido como una respuesta inadecuada ante el estrés, cuya expresión fundamental consiste en un síndrome tridimensional que tiene tres dimensiones características: Un agotamiento físico y/o psicológico, una actitud fría y despersonalizada en el trato con los demás, y un sentimiento de inadecuación ante las tareas que se deben realizar.

Debemos recordar que el término "estrés" proviene de la física y la arquitectura, y se refiere a la fuerza que se aplica a un objeto, que puede deformarlo o romperlo.

En la psicología, el estrés suele hacer referencia a situaciones que implican demandas fuertes para el individuo y que pueden agotar sus recursos de afrontamiento. El término fue importado para la psicología por el fisiólogo canadiense Selye (1956), y desde allí ha sido entendido como: Respuesta-estímulo-interacción. En la actualidad, este último se acepta como el más completo.

El concepto de estrés guarda relación entonces con un desequilibrio entre las demandas del ambiente (extresores externos o internos), y los recursos disponibles del sujeto.

Todo sujeto hace constantes esfuerzos cognitivos y conductuales para manejar adecuadamente las situaciones que se le presentan, por eso no todo el estrés tiene consecuencias negativas; en todo caso, éstas se producen cuando la situación desborda la capacidad de control del sujeto. Este resultado se denomina distrés, a diferencia del estrés positivo o eutrés, que puede ser un buen dinamizador de la actividad laboral.

Volviendo a la temática del "burnout", a partir de la aproximación realizada por Maslach y Jackson, 1981; se han desarrollado colaboraciones (Golembiewski, 1986; Golembiewski y Munzenrider, 1988; Leiter, 1988), en las que se destaca el carácter episódico de un fenómeno descrito a través de fases, aun cuando dichas aportaciones discrepan tanto en la relevancia de unos aspectos sobre otros como en la secuencialidad de la experiencia del "Burnout".

Para Cherniss, 1980, el "burnout" puede ser entendido como una forma de afrontar el éstos, destacando tal vez como aspecto más sugestivo de su teoría, la combinación entre características del entorno laboral y variables personales del trabajador (expectativas, actitudes, etc.), cuya interacción puede generar una sobrecarga capaz de desarrollar respuestas constitutivas de "burnout" como una forma de adaptación defensiva.

A partir de la propuesta anteriormente mencionada, las características personales de quienes desarrollan profesiones orientadas hacia la ayuda a los demás, pueden explicar en buena 
medida su mayor predisposición a terminar "quemados", habida cuenta de su carácter vocacional unido a una actitud demasiado exigente respecto a la realización de la propia tarea en la cual puede darse una excesiva implicación emocional.

El "Burnout", sin embargo, no es patrimonio exclusivo de educadores, trabajadores sociales o profesionales de la salud, sino que por el contrario puede ser descrito en todas aquellas "personas que trabajan con personas".

Algunas profesiones por sus características y el contexto en que se ejercen, tienen mas riesgo de ser estresantes. La enseñanza es una de ellas, lo cual ha dado lugar a numerosos estudios sobre los factores implicados y las vías de intervención (Calvete y Villa, 1999; Fernández Arata, 2002). Además, algunos autores (Calvete y Villa, 1997; Kyriakou, 1989), han identificado las fuentes del estrés mas relevantes de los profesores:

(1) Actitudes negativas por parte de los alumnos.

(2) Problemas de disciplina en el aula.

(3) Malas condiciones de trabajo (número excesivo de alumnos en las aulas y falta de equipo adecuado).

(4) Presiones temporales y sobrecarga de trabajo.

(5) Conflictos entre los profesores.

(6) Cambios rápidos en las demandas de currículo y organización.

Cuando la multitud de estresores encontrados en la situación de enseñanza continua sin esperanza de solución, el patrón de reacciones descritas como "Burnout", daña seriamente la habilidad del profesor para ejecutar su trabajo. Los sentimientos del docente se vuelven negativos, se desarrolla una actitud de cinismo, se pierde la implicación con los alumnos, aumentan las enfermedades físicas y mentales y el absentismo y, en ocasiones el consumo de drogas y el alcohol.

A todo lo anteriormente señalado, se pueden agregar trastornos depresivos, ansiedad, irritabilidad, descenso en la autoestima, sentimientos de indefensión, obesidad, consumo de tabaco, insomnio (consecuencias psicológicas) e hipertensión, úlceras, asma, trastornos coronarios (consecuencias psicofisiológicas).

Queda claro entonces que una de las actividades de mayor compromiso profesional en lo que se refiere a apoyar y ayudar a las personas es la docencia. Sobre el punto, la docencia experimenta presiones constantes de la sociedad para intentar corregir problemas sociales de toda índole, por ejemplo, las drogas, el alcohol, el abuso sexual, entre otros. Inclusive, algunos profesionales abandonan la docencia, "quemados" o "hastiados" por la labor diaria para incorporarse en otras actividades laborales.

En virtud a todo lo señalado, creemos que es de suma urgencia estudiar dicha problemática en los docentes universitarios de nuestro país, toda vez que en su ejercicio profesional los profesores están orientados permanentemente al trato directo con las personas, desarrollando un alto grado de compromiso emocional, que puede en un momento determinado provocar el denominado estrés laboral. 


\section{Problema de investigación}

El presente estudio de tipo descriptivo comparativo pretende responder a las siguientes interrogantes:

1. ¿Presentan los docentes universitarios peruanos, evidencias del llamado síndrome del "quemado" por estrés laboral asistencial?

2. ¿Existen diferencias en los componentes del síndrome del "quemado", en grupos de docentes universitarios, en razón al área de especialidad a la que pertenecen?

3. ¿Existen diferencias en los componentes del síndrome del "quemado", en grupos de docentes universitarios, en razón al sexo, estado civil, práctica de deporte, problemas de salud, condición docente?

4. ¿Existe correlación entre los tres factores del "Burnout" y las variables Años de Servicios en la Universidad y Tiempo se servicios en la profesión?

\section{Justificación}

El síndrome del "quemado" por estrés laboral asistencial o "Burnout", constituye una temática muy importante en la psicología contemporánea y ha sido poco estudiada por los docentes universitarios en nuestro país.

Al conocer comparativamente el síndrome del "quemado" por estrés laboral asistencial en los docentes universitarios, pertenecientes a diversas áreas de especialidad, podremos precisar posibles diferencias que inclusive podrían deberse a otras variables (Facultad de pertenencia, etc.). En todo caso, el conocimiento de esta problemática nos va a permitir formular estrategias de intervención en estos grupos de profesores que constituyen la base de la enseñanza universitaria.

Resulta necesario efectuar los estudios de confiabilidad y validez del MBI-Inventario "Burnout" de Maslach y Jackson.

El estudio va a permitir contar con normas o baremos del MBl, correspondientes a grupos de profesores que hacen docencia universitaria.

\section{Objetivos}

1. Conocer la posible presencia del síndrome del "quemado" por estrés laboral asistencial en grupos de docentes de una universidad nacional, pertenecientes a diversas áreas de especialidad.

2. Detectar las posibles diferencias significativas en el síndrome del "quemado" por estrés laboral asistencial, en virtud a las variables Sexo, Estado Civil, Práctica de Deporte, Problemas de Salud, y Condición Docente.

\section{Hipótesis}

1. El síndrome del "quemado" por estrés laboral asistencial o "burnout" es una respuesta que se presenta en los docentes de una universidad nacional. 
2. Existen diferencias estadísticamente significativas entre los docentes de una universidad nacional, en relación al síndrome del "quemado" por estrés laboral asistencial, en virtud al área de especialidad a la que pertenecen.

3. Existen diferencias estadísticamente significativas entre los docentes de una universidad nacional, en relación al síndrome del "quemado" por estrés laboral asistencial, en virtud al sexo.

4. Existen diferencias estadísticamente significativas entre los docentes de una universidad nacional, en relación al "burnout", en virtud al estado civil.

5. Existen diferencias significativas entre los docentes de una universidad nacional, en relación al síndrome del "quemado" por estrés laboral asistencial, en virtud a la practica de deporte.

6. Existen diferencias significativas entre los docentes de una universidad nacional, en relación al "burnout", en virtud a problemas de salud.

7. Existen diferencias estadísticamente significativas entre los docentes de una universidad nacional en relación al síndrome del "quemado" por estrés laboral asistencial, en virtud a la condición docente.

\section{MÉTODOS}

\section{El diseño de investigación}

Corresponde al descriptivo comparativo, pues se trata en primer lugar de detectar la posible presencia del "Burnout" en los docentes universitarios; y en segundo lugar, de comparar la presencia del síndrome del "quemado" por estrés laboral asistencial, según el área de estudios, sexo, estado civil, práctica de deporte, problemas de salud, y condición docente, respectivamente.

\section{Universo y muestra de investigación}

El Universo estuvo conformado por todos los Docentes de Universidades Nacionales de Lima Metropolitana. Para el presente estudio se ha trabajado con una muestra representativa de 274 casos, la cual fue seleccionada a través de un muestreo no-probabilistico de tipo intencional.

Tabla 1. Composición de la muestra por área académica.

\begin{tabular}{|l|c|c|}
\hline \multicolumn{1}{|c|}{ Área académica } & Frecuencia & Porcentaje \\
\hline Ciencias de la salud & 96 & 35.0 \\
\hline Ciencias básicas & 49 & 17.9 \\
\hline Ingeniería & 43 & 15.7 \\
\hline Educación, Cc. Ss., Letras & 47 & 17.2 \\
\hline Económico - financiera & 39 & 14.2 \\
\hline Total & 274 & 100.0 \\
\hline
\end{tabular}


La muestra estuvo conformada por 274 docentes universitarios que fueron distribuidos en cinco áreas de especialidad. Un 35\% de los docentes examinados pertenecen al área de las ciencias de la salud; mientras que un $14 \%$ corresponden al área de administración-economíacontabilidad, respectivamente.

Tabla 2. Composición de la muestra por facultad.

\begin{tabular}{|l|c|c|}
\hline \multicolumn{1}{|c|}{ FACULTAD } & FRECUENCIA & PORCENTAJE \\
\hline Odontología & 17 & 6.2 \\
\hline Farmacia & 14 & 5.1 \\
\hline M. Veterinaria & 17 & 6.2 \\
\hline Psicología & 20 & 7.3 \\
\hline Medicina Humana & 28 & 10.2 \\
\hline Biología & 16 & 5.8 \\
\hline Matemáticas & 11 & 4.0 \\
\hline Física & 11 & 4.0 \\
\hline Química & 11 & 4.0 \\
\hline Ing. Industrial & 13 & 4.7 \\
\hline Ing. Electrónica & 8 & 2.9 \\
\hline Geología & 11 & 4.0 \\
\hline Ing. de Sistemas & 11 & 4.0 \\
\hline Educación & 21 & 7.7 \\
\hline Ciencias. Sociales & 19 & 6.9 \\
\hline Letras & 7 & 2.6 \\
\hline Economía & 15 & 5.5 \\
\hline Contabilidad & 12 & 4.4 \\
\hline Administración & 12 & 4.4 \\
\hline Total & 274 & 100.0 \\
\hline
\end{tabular}

La mayor proporción de docentes examinados ha sido de las Facultades de Medicina (10.2\%); Educación (7.7\%); y Psicología (7.3\%); mientras que la menor proporción de docentes evaluados ha sido de Letras (2.6\%); y Electrónica (2.9\%), respectivamente. 
Revista de Investigación en Psicología - Vol. 8, N. ${ }^{\circ} 2$

Tabla 3. Composición de la muestra por edad cronológica.

\begin{tabular}{|c|c|c|}
\hline Edad & Frecuencia & Porcentaje \\
\hline 23 & 1 & 0.4 \\
\hline 24 & 2 & 0.7 \\
\hline 26 & 7 & 2.6 \\
\hline 27 & 3 & 1.1 \\
\hline 28 & 1 & 0.4 \\
\hline 29 & 1 & 0.4 \\
\hline 30 & 1 & 0.4 \\
\hline 31 & 5 & 1.8 \\
\hline 32 & 4 & 1.5 \\
\hline 33 & 3 & 1.1 \\
\hline 34 & 2 & 0.7 \\
\hline 35 & 4 & 1.5 \\
\hline 36 & 2 & 0.7 \\
\hline 37 & 1 & 0.4 \\
\hline 38 & 7 & 2.6 \\
\hline 39 & 9 & 3.3 \\
\hline 40 & 23 & 8.4 \\
\hline 41 & 14 & 5.1 \\
\hline 42 & 13 & 4.7 \\
\hline 43 & 11 & 4.0 \\
\hline 44 & 8 & 2.9 \\
\hline 45 & 16 & 5.8 \\
\hline 46 & 6 & 2.2 \\
\hline 47 & 4 & 1.5 \\
\hline 48 & 6 & 2.2 \\
\hline 49 & 6 & 2.2 \\
\hline 50 & 25 & 9.1 \\
\hline 51 & 8 & 2.9 \\
\hline 52 & 16 & 5.8 \\
\hline 53 & 6 & 2.2 \\
\hline 54 & 9 & 3.3 \\
\hline 55 & 9 & 3.3 \\
\hline 56 & 4 & 1.5 \\
\hline 57 & 7 & 2.6 \\
\hline 58 & 2 & 0.7 \\
\hline 59 & 4 & 1.5 \\
\hline 60 & 6 & 2.2 \\
\hline 61 & 4 & 1.5 \\
\hline 62 & 2 & 0.7 \\
\hline 63 & 3 & 1.1 \\
\hline 64 & 3 & 1.1 \\
\hline 65 & 1 & 0.4 \\
\hline 69 & 1 & 0.4 \\
\hline 71 & 1 & 0.4 \\
\hline No responde & 3 & 1.1 \\
\hline Total & 274 & 100.00 \\
\hline
\end{tabular}


En la referencia podemos apreciar que las edades cronológicas de los docentes examinados ha oscilado entre 23 años $(0.4 \%)$, y 71 años $(0.4 \%)$. El mayor porcentaje de docentes ha sido de 50 años (9.2\%), y de 40 años (8.5\%); asimismo, ha habido tres docentes (1.1\%) que no respondieron a esta pregunta.

Tabla 4. Composición de la muestra por sexo.

\begin{tabular}{|l|c|c|}
\hline Sexo & Frecuencia & Porcentaje \\
\hline Varón & 202 & 73.7 \\
\hline Mujer & 72 & 26.3 \\
\hline Total & 274 & 100.0 \\
\hline
\end{tabular}

Tabla 5. Composición de la muestra por Estado Civil.

\begin{tabular}{|l|c|c|}
\hline Estado civil & Frecuencia & Porcentaje \\
\hline Soltero & 59 & 21.5 \\
\hline Casado & 195 & 71.2 \\
\hline Viudo & 5 & 1.8 \\
\hline Divorciado & 14 & 5.1 \\
\hline Separado & 1 & 0.4 \\
\hline Total & 274 & 100.00 \\
\hline
\end{tabular}

En la referenda, podemos apreciar que el $71.2 \%$ de docentes son casados, un $21.5 \%$ son solteros, y un $5.1 \%$ son divorciados. Finalmente, existe un $1.8 \%$ viudos y un $0.4 \%$ de separados.

Tabla 6. Composición de la muestra por práctica de deporte.

\begin{tabular}{|l|c|c|}
\hline Practica deporte & Frecuencia & Porcentaje \\
\hline Sí & 122 & 44.5 \\
\hline No & 152 & 55.5 \\
\hline Total & 274 & 100.0 \\
\hline
\end{tabular}

En la referenda podemos apreciar que el $55.5 \%$ de docentes universitarios examinados no practican deporte, en tanto que el $44.5 \%$ restante sí lo hace. 
Tabla 7. Deporte que practica.

\begin{tabular}{|c|c|c|}
\hline Deporte & Frecuencia & Porcentaje \\
\hline 0 & 152 & 55.5 \\
\hline 1 & 5 & 1.8 \\
\hline 2 & 35 & 12.8 \\
\hline 3 & 47 & 17.2 \\
\hline 4 & 2 & 0.7 \\
\hline 5 & 9 & 3.3 \\
\hline 6 & 4 & 1.5 \\
\hline 7 & 2 & 0.7 \\
\hline 8 & 3 & 1.1 \\
\hline 9 & 15 & 5.5 \\
\hline Total & $\mathbf{2 7 4}$ & $\mathbf{1 0 0 . 0}$ \\
\hline
\end{tabular}

En la referencia podemos apreciar que de los docentes que practican deporte, el mayor porcentaje practican fútbol (17.2\%) y fulbito (12.8\%), mientras que el menor porcentaje practican aeróbicos $(0.4 \%)$ y artes marciales $(0.4 \%)$, respectivamente.

\section{Variables de estudio}

- Variables independientes asignadas: Área de especialidad, facultad de procedencia, sexo, estado civil, práctica de deporte, problemas de salud y condición docente.

- Variables dependientes: Cansancio emocional, despersonalización y realización personal.

\section{Técnicas e instrumentos de recolección de datos}

En el presente estudio se ha utilizado el MBI-Inventario "Burnout" de C. Maslach y S.E. Jackson, el cual comprende una escala tridimensional, y a partir de ella se han definido las siguientes escalas o variables del sujeto "quemado", las cuales se indican a continuación:

1. Cansancio emocional (CE), compuesta por nueve ítemes. Sus elementos describen los sentimientos de una persona exhausta por el propio trabajo; el elemento con mayor saturación contiene una expresión clara de dicho sentimiento: "Me siento emocionalmente agotado por mi trabajo".

2. Despersonalización (DP), compuesta por cinco elementos. Los elementos de esta escala describen una respuesta impersonal y fría hacia los receptores de los servicios o cuidados del personal. Tanto en esta como en la escala anterior, los profesionales con puntuaciones altas revelan grados elevados de vivencia del síndrome del estrés laboral asistencial. Como algunos de sus elementos presentan pequeñas saturaciones en la otra escala, se observan pequeñas correlaciones entre ambas dimensiones. Es decir, aunque son medidas separadas, están correlacionadas y se presentan como aspectos del síndrome. 
3. Realización Personal (RP), compuesta por ocho itemes. Esta escala contiene elementos que describen sentimientos de competencia y éxito en el trabajo propio con personas. En contraste con las otras dos escalas, las puntuaciones bajas son indicativas del síndrome; pero es independiente de ellas y sus elementos no tienen pesos negativos en ellas. La variable puede ser considerada como lo opuesto al cansancio emocional y a la despersonalización. Sin embargo, se observan correlaciones bajas con las otras dos escalas.

\section{Análisis psicométrico}

Tabla 8. Análisis psicométrico de la Escala de Cansancio Emocional del MBI. Inventario"Burnout" de Maslach.

\begin{tabular}{|c|c|c|c|}
\hline Ítem & $\mathbf{M}$ & D.E & rit \\
\hline CE1 & 1.98 & 1.74 & 0.73 \\
\hline CE2 & 2.75 & 1.71 & 0.55 \\
\hline CE3 & 1.77 & 1.72 & 0.60 \\
\hline CE6 & 1.71 & 1.94 & 0.46 \\
\hline CE8 & 1.24 & 1.75 & 0.71 \\
\hline CE13 & 1.10 & 1.59 & 0.52 \\
\hline CE14 & 2.56 & 2.12 & 0.45 \\
\hline CE16 & 1.34 & 1.61 & 0.56 \\
\hline CE20 & 0.57 & 1.19 & 0.58 \\
\hline \multicolumn{4}{|c|}{ Alfa de Crombach $=\mathbf{0 . 8 5}$} \\
\hline
\end{tabular}

$$
\mathrm{N}=274
$$

Los valores obtenidos nos revelan que los itemes presentan correlaciones ítem-test corregidas que fluctúan entre 0.45 para el caso del ítem CE14; y 0.73 para el caso del ítem 1, respectivamente. Los resultados corroboran que los itemes son consistentes entre si. Finalmente, el análisis de la confiabilidad por consistencia interna indica un coeficiente alfa de 0.85 , lo que nos permite inferir la existencia de puntajes confiables.

Tabla 9. Análisis psicométrico de la Escala de Despersonalización del MBI Inventario "Burnout" de Maslach.

\begin{tabular}{|c|c|c|c|}
\hline Ítem & M & D.E. & rit \\
\hline DES5 & 0.88 & 1.31 & 0.49 \\
\hline DES10 & 1.16 & 1.76 & 0.43 \\
\hline DES11 & 1.19 & 1.63 & 0.45 \\
\hline DES15 & 1.81 & 2.17 & 0.22 \\
\hline DES22 & 1.19 & 1.41 & 0.40 \\
\hline \multicolumn{4}{|c|}{ Alfa de Crombach $=\mathbf{0 . 6 6}$} \\
\hline
\end{tabular}

$$
\mathrm{N}=274
$$


Los resultados obtenidos en la variable Despersonalización nos indica que los itemes presentan correlaciones ítem-test corregidas que fluctúan entre 0.22 para el caso del ítem D15; y de 0.49 para el caso del ítem 5. Los valores revelan que los ítemes son consistentes entre sí. El análisis de confiabilidad por consistencia interna indica un coeficiente alfa de 0.66 , lo que nos permite inferir la existencia de puntajes confiables.

Tabla 10. Análisis psicométrico de la Escala de Realización Personal del MBI Inventario "burnout" de Maslach.

\begin{tabular}{|c|c|c|c|}
\hline Item & M & D.E. & rit \\
\hline RELPER 4 & 4.23 & 1.99 & 0.46 \\
\hline RELPER 7 & 3.96 & 1.89 & 0.49 \\
\hline RELPER 9 & 4.77 & 1.74 & 0.55 \\
\hline RELPER 12 & 5.04 & 1.39 & 0.37 \\
\hline RELPER 17 & 4.05 & 2,05 & 0.47 \\
\hline RELPER 18 & 4.98 & 1.59 & 0.58 \\
\hline RELPER 19 & 4.62 & 1.79 & 0.42 \\
\hline RELPER 21 & 4.36 & 1.91 & 0.55 \\
\hline \multicolumn{4}{|c|}{ Alfa de Crombach $=\mathbf{0 . 7 8}$} \\
\hline
\end{tabular}

$\mathrm{N}=274$

Los valores obtenidos nos revelan que los itemes presentan correlaciones ítem-test corregidas que fluctúan entre 0.37 para el ítem RP12; y de 0.58 para el ítem RP18, respectivamente. Los puntajes corroboran que los itemes son consistentes entre si. Asimismo, el análisis de confiabilidad por consistencia interna indica un coeficiente alfa de 0.78 , lo que nos permite inferir la existencia de puntajes confiables.

Tabla 11. Análisis Factorial Exploratorio del MBI Inventario "Burnout" de Maslach.

\begin{tabular}{|c|c|c|c|}
\hline Variables & Media & $\begin{array}{c}\text { Realización } \\
\text { típica }\end{array}$ & Factor 1 \\
\hline Cansancio emocional & 15.02 & 10.42 & 0.79 \\
\hline Despersonalización & 6.23 & 5.32 & 0.81 \\
\hline Realización personal & 35.99 & 9.07 & -0.60 \\
\hline \multicolumn{3}{|c|}{ Varianza explicada } & 54.512 \\
\hline $\begin{array}{l}\text { Medida de aplicación muestral de Kaiser-Meyer-Olkin. }=0.87 \\
\text { Prueba de esfericidad de Bartlett Chi-cuadrado aproximado }= \\
\text { 85.84 G.L. }=3\end{array}$.sig. =000 \\
\hline
\end{tabular}

$\mathrm{N}=274$ 
Los valores obtenidos nos revelan que existe un solo factor bipolar de orden superior que tiene una varianza que explica el $54.5 \%$ de la varianza total. En suma, entre estos tres puntajes existe esta asociación, lo cual corrobora la presencia del Síndrome del "quemado" por estrés laboral asistencial o "burnout".

\section{Técnicas de procesamiento y análisis de datos}

El análisis estadístico ha comprendido los siguientes aspectos:

1. Análisis descriptivo y exploratorio para describir de manera detallada la distribución de la muestra examinada; en tal sentido, se han calculado para las variables estudiadas: las frecuencias, los porcentajes, las medias o promedios aritméticos, las desviaciones Standard, así como los análisis de tallos y hojas y las pruebas de ajuste a la curva normal de Kolmogorov-Smirnov para los puntajes de las escalas de las pruebas.

2. Análisis psicométrico del MBI-Inventario "burnout" de Maslach y Jackson, que incluye: el análisis de itemes con el cómputo de las correlaciones ítem-test; el estudio de la validez de constructo, que incluye el desarrollo del análisis factorial exploratorio, y un análisis factorial confirmatorio; y la confiabilidad, cálculo de los coeficientes Alfa de Crombach.

3. Análisis inferencial para la contestación de hipótesis, para lo cual se ha ejecutado: el análisis de varianza de dos factores por áreas de especialidad; sexo; estado civil; practica de deporte; problemas de salud; y condición docente.

\section{RESULTADOS}

Análisis descriptivo

Tabla 12. Análisis de la normalidad de los puntajes MBI-Inventario "burnout" de Maslach.

\begin{tabular}{|c|c|c|c|}
\hline Variables & M & D.E. & Z de Kolmogorov-Smirnov \\
\hline Cansancio emocional & 15.02 & 10.42 & $2.07 *$ \\
\hline Despersonalización & 6.23 & 5.32 & $2.00 *$ \\
\hline Realización personal & 35.99 & 9.07 & $2.08 *$ \\
\hline
\end{tabular}

$$
\mathrm{N}=274
$$

En la referenda, podemos apreciar que en el MBI-Inventario de "burnout" de Maslach, los resultados no se aproximan a la distribución normal, en tal sentido, en los tres factores, los valores son menores a 0.05 (2-Tailed $\mathrm{P}: \mathrm{CE}=.0004$; $\mathrm{DESP}=.0007$; y $\mathrm{RP}=.0003$ ), por lo que debe trabajarse la investigación con estadísticos no-paramétricos sobre la base de rangos. 


\section{Análisis del contraste de hipótesis}

H1: El síndrome del "burnout" es una respuesta al estrés profesional que se está presentando en los docentes de una universidad nacional.

En virtud a los resultados del análisis factorial exploratorio que se presenta en la tabla 11, podemos apreciar que entre los tres factores del "burnout", existe una asociación que revela la existencia de un solo factor bipolar de orden superior que tiene una varianza que explora el $54.5 \%$ de la varianza total, lo cual corrobora la presencia del Síndrome en los docentes universitarios.

Asimismo, y teniendo en cuenta los valores obtenidos que se detallan en la tabla 20, podemos apreciar que un $45.4 \%$ de Docentes se encuentran en la fase inicial del "Burnout"; un 7.3\% se hallan en el nivel intermedio, y un $46.3 \%$ se encuentran en la fase final, hallazgos que permiten ratificar la Hipótesis 1.

H2: Existen diferencias estadísticamente significativas entre los docentes de una Universidad Nacional, en relación al síndrome del "quemado" por estrés laboral asistencial, en virtud al área de especialidad a la que pertenecen.

Tabla 13. Estudio comparativo del MBI por Área de especialidad.

\begin{tabular}{|l|c|c|c|c|c|c|}
\hline \multirow{2}{*}{ Variable } & $\begin{array}{c}\text { Área 1 } \\
\mathbf{N = 9 6}\end{array}$ & $\begin{array}{c}\text { Área 2 } \\
\mathbf{N = 4 9}\end{array}$ & $\begin{array}{c}\text { Área 3 } \\
\mathbf{N = 4 3}\end{array}$ & $\begin{array}{c}\text { Área 4 } \\
\mathbf{N = 4 7}\end{array}$ & $\begin{array}{c}\text { Área 5 } \\
\mathbf{N = 3 9}\end{array}$ & \multirow{2}{*}{$\mathbf{F}$} \\
\cline { 2 - 6 } & $\mathbf{M}$ & $\mathbf{M}$ & $\mathbf{M}$ & $\mathbf{M}$ & $\mathbf{M}$ & \\
\hline Cansancio emocional & 17.19 & 13.27 & 14.53 & 12.06 & 15.97 & $2.49^{*}$ \\
\hline Despersonalización & 6.61 & 5.80 & 6.67 & 5.98 & 5.64 & 0.42 \\
\hline Realización personal & 38.02 & 34.10 & 34.21 & 36.85 & 34.31 & $2.65^{*}$ \\
\hline
\end{tabular}

$* \mathrm{p}<.05$

La tabla nos permite apreciar, en primer lugar, la existencia de diferencias en el área de cansancio emocional entre los docentes examinados en razón al área de especialidad de procedencia, sin embargo dichas diferencias no son significativas, son ficticias, es decir, aparecen en F, pero al dividirse o compararse por pares de grupos, no se marcan las diferencias (test de Scheffe).

En segundo lugar, la tabla nos revela que no existen diferencias significativas entre los docentes examinados en virtud a su área de especialidad, respecto a la variable despersonalización.

En tercer lugar, la tabla nos permite apreciar la existencia de diferencias en la variable Realización Personal entre los docentes universitarios pertenecientes a diferentes áreas de especialidad, sin embargo, tales diferencias no son significativas, son ficticias, tal y como ha ocurrido con la variable cansancio emocional. 
H3: Existen diferencias estadísticamente significativas entre los docentes de una universidad nacional, en relación al síndrome del "quemado" por estrés laboral asistencial, en virtud al sexo.

Tabla 14. Estudio comparativo del MBI por sexo.

\begin{tabular}{|c|c|c|c|c|c|}
\hline \multirow{2}{*}{ Variables } & \multicolumn{2}{|c|}{$\begin{array}{c}\text { Hombres } \\
\text { N= 202 }\end{array}$} & \multicolumn{2}{c|}{$\begin{array}{c}\text { Mujeres } \\
\text { N = 72 }\end{array}$} & \multirow{2}{*}{ Z } \\
\cline { 2 - 5 } & $\mathbf{M}$ & $\mathbf{D : E :}$ & $\mathbf{M}$ & D.E: & \\
\hline Cansancio emocional & 14.72 & 10.31 & 15.86 & 10.76 & -0.80 \\
\hline Despersonalización & 6.57 & 5.46 & 5.26 & 4.83 & 1.80 \\
\hline Realización personal & 36.15 & 8.92 & 35.56 & 9.51 & 0.48 \\
\hline
\end{tabular}

La tabla nos permite apreciar que no existen diferencias significativas entre docentes hombres y mujeres respecto a la variable cansancio emocional.

Los valores obtenidos permiten apreciar que no existen diferencias significativas entre docentes hombres y mujeres en relación a la variable despersonalización.

Los resultados arrojan que no existen diferencias significativas entre docentes hombres y mujeres en relación a la variable realización personal

H4: Existen diferencias estadísticamente significativas entre los docentes de una Universidad Nacional, en relación al "burnout", en virtud al Estado Civil.

Tabla 15. Estudio Comparativo del MBI por Estado Civil.

\begin{tabular}{|c|c|c|c|c|c|}
\hline \multirow{2}{*}{ Variables } & \multicolumn{2}{|c|}{$\begin{array}{c}\text { Solteros } \\
\text { N= 59 }\end{array}$} & \multicolumn{2}{c|}{$\begin{array}{c}\text { Casados } \\
\text { N= 195 }\end{array}$} & \multirow{2}{*}{ Z } \\
\cline { 2 - 5 } & M & D.E. & M & D.E. & \\
\hline Cansancio emocional & 15.15 & 9.45 & 14.94 & 10.64 & 0.14 \\
\hline Despersonalización & 6.03 & 5.29 & 6.25 & 5.25 & -0.27 \\
\hline Realización personal & 35.20 & 9.25 & 36.03 & 9.10 & -0.61 \\
\hline
\end{tabular}

Los valores obtendos permiten apreciar que no existen diferencias significativas entre los docentes solteros y casados en razón a la variable Cansancio Emocional.

Los resultados obtenidos permiten apreciar que no existen diferencias significativas entre los docentes solteros y casados en razón a la variable Despersonalización. 
Los resultados obtenidos revelan que no existen diferencias significativas entre los docentes solteros y casados en razón a la variable realización Personal.

Los resultados arrojan que no existen diferencias significativas entre docentes hombres y mujeres en relación a la variable Realización Personal.

H5: Existen diferencias estadísticamente significativas entre los docentes de una universidad nacional, en relación al "burnout", en virtud al Estado Civil.

Tabla 16. Estudio Comparativo del MBI por práctica del deporte.

\begin{tabular}{|c|c|c|c|c|c|}
\hline \multirow[t]{2}{*}{ Variables } & \multicolumn{2}{|c|}{ Solteros N $=122$} & \multicolumn{2}{|c|}{$\begin{array}{r}\text { Casados } \\
\mathbf{N}=152\end{array}$} & \multirow[t]{2}{*}{$\mathbf{Z}$} \\
\hline & $\mathbf{M}$ & D:E: & $\mathbf{M}$ & D:E: & \\
\hline Cansancio Emocional & 13.99 & 9.42 & 15.84 & 11.13 & -1.46 \\
\hline Despersonalización & 6.60 & 5.28 & 5.93 & 5.35 & 1.03 \\
\hline Realización Personal & 35.50 & 8.56 & 36.39 & 9.46 & -0.81 \\
\hline
\end{tabular}

Los valores obtenidos permiten apreciar que no existen diferencias significativas entre los docentes solteros y casados en razón a la variable Cansancio Emocional.

Los resultados obtenidos permiten apreciar que no existen diferencias significativas entre los docentes solteros y casados en razón a la variable despersonalización.

Los resultados obtenidos revelan que no existen diferencias significativas entre los docentes solteros y casados en razón a la variable realización personal

H6: Existen diferencias significativas entre los docentes de una universidad nacional, en relación al "burnout", en virtud a problemas de salud.

Tabla 17. Estudio comparativo del MBI por problemas de salud.

\begin{tabular}{|c|c|c|c|c|c|}
\hline \multirow{2}{*}{ Variables } & \multicolumn{2}{|c|}{$\begin{array}{c}\text { Sí } \\
\mathbf{N}=77\end{array}$} & \multicolumn{2}{|c|}{$\begin{array}{c}\text { No } \\
\mathbf{N}=197\end{array}$} & \multirow[t]{2}{*}{$\mathbf{Z}$} \\
\hline & M & D.E. & M & D.E. & \\
\hline Cansancio emocional & 19.48 & 11.23 & 13.27 & 9.57 & $4.59 * * *$ \\
\hline Despersonalización & 7.09 & 6.09 & 5.89 & 4.96 & 1.68 \\
\hline Realización personal & 35.10 & 9.36 & 36.34 & 8.95 & -1.01 \\
\hline
\end{tabular}

$* * * \mathrm{p}<.001$ 
Los valores obtenidos nos revelan la existencia de diferencias significativas en la variable cansancio emocional entre los docentes que presentan problemas de salud, respecto de los que no los presentan.

Los resultados obtenidos nos revelan que no existen diferencias significativas en la variable despersonalización entre los docentes que presentan problemas de salud, respecto de los que no los presentan.

Los valores obtenidos revelan que no existen diferencias significativas entre los docentes que presentan problemas de salud respecto de los que no los presentan, en la variable realización personal.

H7: Existen diferencias estadísticamente significativas entre los Docentes de una universidad nacional, en relación al síndrome del "quemado" por estrés laboral asistencial, en virtud a la condición docente.

Tabla 18. Estudio comparativo del MBI por Nombrados-Contratados.

\begin{tabular}{|l|c|c|c|c|c|}
\hline \multirow{2}{*}{\multicolumn{1}{c|}{ Variables }} & \multicolumn{2}{|c|}{ Sí } & \multicolumn{2}{c|}{ No } & \multirow{2}{*}{ N } \\
\cline { 2 - 5 } & $\mathrm{M}$ & D.E. & $\mathrm{M}$ & D.E. & \\
\hline Cansancio emocional & 14.85 & 10.60 & 15.81 & 9.62 & -50.7 \\
\hline Despersonalización & 5.94 & 5.16 & 7.64 & 5.88 & $-2.00^{*}$ \\
\hline Realización personal & 36.34 & 8.70 & 34.30 & 10.60 & -1.41 \\
\hline
\end{tabular}

$* \mathrm{p}<.05$

Los resultados obtenidos revelan que no existen diferencias significativas entre los docentes nombrados y contratados en relación a la variable cansancio emocional.

Los valores obtenidos revelan que existen diferencias significativas entre los docentes nombrados y contratados en la variable despersonalización, es decir, los contratados se encuentran despersonalizados frente a los nombrados.

Los valores obtenidos revelan que no existen diferencias significativas entre los docentes nombrados y contratados en la variable realización personal. 


\section{Análisis complementarios}

Tabla 19. Correlaciones entre el "burnout" y el tiempo de servicios en la profesión y entre el "burnout" y el tiempo de servicios como docente.

\begin{tabular}{|l|c|c|}
\hline \multicolumn{1}{|c|}{ Variables } & $\begin{array}{c}\text { Tiempo de servicios } \\
\text { en la profesión }\end{array}$ & $\begin{array}{c}\text { Tiempo de servicios } \\
\text { como docente }\end{array}$ \\
\hline Cansancio emocional & $-0.16^{*}$ & $-0.13^{\prime \prime}$ \\
\hline Despersonalización & $-0.21^{* *}$ & $-0.14^{*}$ \\
\hline Realización personal & $0.14^{*}$ & $0.12^{*}$ \\
\hline
\end{tabular}

$* \mathrm{p}<.05$

$\mathrm{N}=274$

Los valores obtenidos revelan que a mayor tiempo de servicios en la profesión: Menor cansancio emocional, menor despersonalización y mayor realización personal, y viceversa. Asimismo, a mayor tiempo de servicios como docente: menor cansancio emocional; menor despersonalización; y mayor realización personal y viceversa.

En virtud a los hallazgos obtenidos, pareciera que el "burnout" es una respuesta objetiva de la situación actual del docente universitario.

Tabla 20. Etapas del "burnout" por áreas de estudio según la tabla de Golembiewsky.

\begin{tabular}{|l|c|c|c|c|c|c|}
\hline Etapa & $\begin{array}{c}\text { Ciencias } \\
\text { de la } \\
\text { salud }\end{array}$ & $\begin{array}{c}\text { Ciencias } \\
\text { básicas }\end{array}$ & Ingeniería & $\begin{array}{c}\text { Educación, } \\
\text { Cc. Ss., } \\
\text { Letras }\end{array}$ & $\begin{array}{c}\text { Económico- } \\
\text { financiero }\end{array}$ & Total \\
\hline Etapa I & 3 & 6 & 3 & 3 & 0 & 15 \\
\hline Etapa II & 22 & 21 & 14 & 22 & 15 & 94 \\
\hline Etapa III & 7 & 1 & 1 & 4 & 5 & 18 \\
\hline Etapa IV & 7 & 1 & 1 & 3 & 1 & 13 \\
\hline Etapa V & 0 & 2 & 2 & 3 & 0 & 7 \\
\hline Etapa VI & 45 & 16 & 19 & 12 & 16 & 108 \\
\hline Etapa VII & 1 & 0 & 0 & 0 & 1 & 2 \\
\hline Etapa VIII & 11 & 2 & 3 & 0 & 1 & 17 \\
\hline Total & 96 & 49 & 43 & 47 & 39 & 274 \\
\hline
\end{tabular}

Chi cuadrado de Pearson $=46.32$ G.L. $=28 \mathrm{p}<.05$

Los resultados obtenidos permiten apreciar según la tabla de Golembiesky que un $45.4 \%$ de Docentes se encuentran en la Fase inicial del "burnout"; un $7.3 \%$ se encuentran en la fase intermedia; en tanto que un $46.3 \%$ se encuentran en la fase final, es decir, ya tienen "burnout". 
Cabe mencionar que según Golembiesky, el paso del nivel IV hacia delante es mas rápido, por lo que dicha proporci6n puede incrementarse al $53.6 \%$, respectivamente. Este resultado guarda relaci6n con la primera interrogante planteada. Asimismo, podemos apreciar que un 59\% de Docentes de las Ciencias de la Salud manifiestan "burnout" en los niveles VI, VII, y VIII, frente al 25\% de Docentes de Letras, Educación, y Derecho, lo cual nos plantea diferencias cualitativas importantes de tener en cuenta.

Tabla 21. Etapas del "burnout" por sexo según la tabla de Golembiewsky.

\begin{tabular}{|l|c|c|c|}
\hline \multicolumn{1}{|c|}{ Etapa } & Varón & Mujer & total \\
\hline Etapa I & 10 & 5 & 15 \\
\hline Etapa II & 71 & 23 & 94 \\
\hline Etapa III & 14 & 4 & 18 \\
\hline Etapa IV & 9 & 4 & 13 \\
\hline Etapa V & 6 & 1 & 7 \\
\hline Etapa VI & 79 & 29 & 108 \\
\hline Etapa VII & 1 & 1 & 2 \\
\hline Etapa VIII & 12 & 5 & 17 \\
\hline Total & 202 & 72 & 274 \\
\hline
\end{tabular}

Chi-cuadrado de Pearson $=2.04$ G. L. $=7 \mathrm{p}>.05$

Los valores obtenidos permiten apreciar que un $45 \%$ de docentes varones presentan niveles VI, VII, y VIII en la Tabla del "burnout", en tanto que un $48 \%$ de mujeres también lo presentan. En suma, se encuentra equilibrada la presencia del Síndrome del "quemado" por Stress Laboral Asistencial en los docentes universitarios, en función a la variable Sexo.

Tabla 22. Etapas del "burnout" por Estado Civil según la tabla de Golembiewsky.

\begin{tabular}{|l|c|c|c|c|c|c|}
\hline \multicolumn{1}{|c|}{ Etapa } & Soltero & Casado & Viudo & Divorciado & Separado & Total \\
\hline Etapa 1 & 3 & 12 & 0 & 0 & 0 & 15 \\
\hline Etapa 11 & 23 & 64 & 3 & 4 & 0 & 94 \\
\hline Etapa III & 3 & 12 & 1 & 2 & 0 & 18 \\
\hline Etapa IV & 2 & 11 & 0 & 0 & 0 & 13 \\
\hline Etapa V & 1 & 6 & 0 & 0 & 0 & 7 \\
\hline Etapa VI & 24 & 74 & 1 & 8 & 1 & 108 \\
\hline Etapa VII & 1 & 1 & 0 & 0 & 0 & 2 \\
\hline Etapa III & 2 & 15 & 0 & 0 & 0 & 17 \\
\hline Total & 59 & 195 & 5 & 14 & 1 & 274 \\
\hline
\end{tabular}

Chi-cuadrado de Pearson $=14.94$ G.L. $=28$ p $>.05$ 
Los resultados obtenidos permiten apreciar que los docentes universitarios examinados presentan un equilibrio proporcional del $46 \%$ (solteros y casados), en la presencia de los niveles VI, VII, y VIII, del "burnout", en razón a la variable estado civil. En vista del reducido número de docentes que manifiestan otras modalidades de estado civil, estas no se han tomado en cuenta en el análisis.

Tabla 23. Etapas del "burnout" por práctica de deporte, según la tabla de Golembiewsky.

\begin{tabular}{|l|c|c|c|}
\hline \multicolumn{1}{|c|}{ Etapa } & Sí & No & Total \\
\hline Etapa 1 & 6 & 9 & 15 \\
\hline Etapa 11 & 45 & 49 & 94 \\
\hline Etapa III & 5 & 13 & 18 \\
\hline Etapa IV & 8 & 5 & 13 \\
\hline Etapa V & 3 & 4 & 7 \\
\hline Etapa VI & 50 & 58 & 108 \\
\hline Etapa VII & 0 & 2 & 2 \\
\hline Etapa VIII & 5 & 12 & 17 \\
\hline Total & 122 & 152 & 274 \\
\hline
\end{tabular}

Chi-cuadrado de Pearson $=7.44$ G. L. $=7 \mathrm{p}>.05$

Los hallazgos obtenidos nos revelan que en razón a la variable Práctica de Deporte, los Docentes Universitarios manifiestan un equilibrio proporcional de la presencia del "burnout" en los niveles VI, VII, y VIIL, del $45 \%$ y $47 \%$ (practican y no practican deporte), es decir, los valores se encuentran equilibrados.

Tabla 24. Etapas del "burnout" por problemas de salud según la tabla de Golembiewsky.

\begin{tabular}{|l|c|c|c|}
\hline \multicolumn{1}{|c|}{ Etapa } & Sí & No & Total \\
\hline Etapa 1 & 2 & 13 & 15 \\
\hline Etapa II & 18 & 76 & 94 \\
\hline Etapa III & 5 & 13 & 18 \\
\hline Etapa IV & 4 & 9 & 13 \\
\hline Etapa V & 2 & 5 & 7 \\
\hline Etapa VI & 41 & 67 & 108 \\
\hline Etapa VII & 1 & 1 & 2 \\
\hline Etapa VIII & 4 & 13 & 17 \\
\hline Total & 77 & 197 & 274 \\
\hline
\end{tabular}

Chi-cuadrado de Pearson $=11.24$ G. L. $=7 \mathrm{p}>.05$ 
Los resultados obtenidos permiten apreciar que en razón a la variable Problemas de Salud, los docentes universitarios examinados evidencian diferencias porcentuales del $60 \%$ (los que presentan problemas de salud), frente al $41 \%$ (los que no presentan problemas de salud). En suma, al parecer en los docentes universitarios, la presencia del Síndrome del "quemado" por stress laboral asistencial guarda relación con el estado de salud.

Tabla 25. Etapas del "burnout" por Condición Docente: Nombrado / Contratado,según la tabla de Golembiewsky.

\begin{tabular}{|l|c|c|c|}
\hline \multicolumn{1}{|c|}{ Etapa } & N & C & Total \\
\hline Etapa I & 14 & 1 & 15 \\
\hline Etapa II & 78 & 16 & 94 \\
\hline Etapa III & 17 & 1 & 18 \\
\hline Etapa IV & 10 & 3 & 13 \\
\hline Etapa V & 6 & 1 & 7 \\
\hline Etapa VI & 88 & 20 & 108 \\
\hline Etapa VII & 2 & 0 & 2 \\
\hline Etapa VIII & 12 & 5 & 17 \\
\hline Total & 227 & 47 & 274 \\
\hline
\end{tabular}

Chi-cuadrado de Pearson $=5.58$ G. L. $=7 \mathrm{p}>.05$

Los valores obtenidos nos revelan que en la variable condición docente: nombrado-contratado, los profesores universitarios contratados presentan un $53 \%$ de "burnout" correspondiente a los niveles VI, VII, y VIII, a diferencia de los nombrados que lo presentan en una proporción del $37 \%$, respectivamente.

\section{CONCLUSIONES}

Los resultados obtenidos en el presente estudio nos han permitido llegar a las siguientes conclusiones:

1. Los docentes de la Universidad Nacional Mayor de San Marcos, presentan evidencias del llamado síndrome del "quemado" por estrés laboral asistencial. Por una parte, el análisis factorial exploratorio revela que entre los tres factores existe un solo factor bipolar de orden superior que tiene una varianza que explora el $54.5 \%$ de la varianza total y que corrobora la presencia del "burnout" en los docentes universitarios. De otro lado, el $46.3 \%$ de los profesores tienen "burnout" en niveles significativos o están en la fase final; $7.3 \%$ se encuentran en la fase intermedia y próximos a avanzar a la fase final; y un $46.4 \%$ se encuentran en una fase inicial cuyo desarrollo es lento. Esta conclusión estaría dando respuesta a la hipótesis 1. 
2. El análisis cualitativo permite apreciar diferencias en la presencia del "Burnout" (fase final), según área de especialidad, entre los docentes pertenecientes al área de ciencias de la salud (59\%), respecto de los profesores pertenecientes al área de letras, educaci6n, derecho (25\%), y a los del área básica (37\%), es decir, los primeros presentan niveles significativos del síndrome del "quemado" por estrés laboral asistencial, frente a los segundos. Las otras dos áreas de especialidad (Ingenierías y Económico-Financiera), presentan niveles promedios de "burnout'. Esta conclusión da respuesta a la segunda interrogante y permite ratificar la hipótesis 2 .

3. No existen diferencias significativas en las etapas del "burnout", en relación a la variable sexo. Los hallazgos nos indican un porcentaje de $45 \%$ en los varones y $49 \%$ en las mujeres, conclusión que permite rechazar la hipótesis alternativa 3.

4. No existen diferencias en las etapas del "burnout", en relación a la variable estado civil, lo cual nos indica un porcentaje de $46 \%$ para los solteros; y $46 \%$ para los casados, conclusión que nos permite rechazar la hipótesis alternativa 4 .

5. No existen diferencias en las etapas del "burnout", en relación a la variable Práctica de deporte, la cual nos indica un porcentaje de $45 \%$ para los que practican y $47 \%$ para los que no practican, conclusión que nos permite rechazar la hipótesis alternativa 5.

6. Existen diferencias cualitativas en las etapas del "burnout", en relación a la variable problemas de salud, la cual nos indica un porcentaje de $60 \%$ para los que presentan problemas de salud, frente a un $41 \%$ de docentes que no presentan problemas de salud, lo cual nos permite ratificar la hipótesis 6 .

7. Existen diferencias cualitativas en las etapas del "burnout", en relación a la variable condición docente, la cual nos indica un porcentaje de $37 \%$ para los docentes nombrados, frente a un 53\% para los docentes contratados, estos últimos estarían evidenciando un síndrome del "quemado" por estrés laboral asistencial que podría calificarse de significativo, hallazgo que nos permite ratificar la hipótesis 7.

8. A mayor tiempo de servicios docentes, se infieren: Menor cansancio emocional, menor despersonalización y mayor realización personal, y viceversa.

9. A mayor tiempo en la profesión se detectan: Menor cansancio emocional, menor despersonalización y mayor realización personal, y viceversa.

10. Los docentes que tienen problemas de salud tienen mayor cansancio emocional.

11. Los docentes que tienen problemas de salud manifiestan una mayor despersonalización.

12. Cabe mencionar que en el tratamiento estadístico de los resultados, hemos encontrado pequeñas diferencias ficticias en la variables cansancio emocional y realización personal entre los docentes examinados en razón al área de especialidad a la que pertenecen, pero que al dividirse o compararse por pares de grupos, mediante el test de Scheffe, no se marcan dichas diferencias, al parecer debido a que los grupos de sujetos no son iguales en numero. 


\section{SUGERENCIAS}

En el afán de conocer esta problemática a nivel nacional, se debe promover, no solo estudios de replicación de la presente investigación; sino la realización de otros estudios considerando muestras de docentes de universidades nacionales y particulares de todo el Perú, así como también la inclusión de variables importantes que enriquezcan nuevos proyectos como la ansiedad-estado y la ansiedad-rasgo, entre otros.

Se debe propiciar la realización de estudios multidisciplinarios que puedan darle una mayor cobertura de acción a los hallazgos, especialmente de los pedagogos.

Proponer algunos programas que incluyan algunos antídotos tales como:

- Implicarse en actividades externas al trabajo.

- Intensificar y revalorizar las relaciones personales y familiares.

- Tener tiempo y espacio para el juego y la recreación.

- $\quad$ No postergar los periodos de vacaciones.

- Desarrollar actividad física, deportiva y recreacional.

- Realizar una psicoterapia focalizada en el problema para prevenir una cronicidad del Síndrome.

\section{REFERENCIAS BIBLIOGRÁFICAS}

1. Freudenberger, H.J. (1974). "Staff burnout". Journal of Social Issues, 30,159-165.

2. Casas Hilari, Miguel (2002). Cuando querer no es poder. Reportaje. BDN Training. Allbeco.

3. Edelwich, J. y Brodsky, A. (1980). Burnout: Stages of disillisuoment in the helping professions. New York: Human Sciences Press.

4. Guerrero Barona, Eloisa (1998). Burnout o desgase fisico y afrontamiento del estrés en el profesorado universitario. Tesis doctoral no publicada. Facultad de Educación. Universidad de Extremadura. España.

5. Maslach, C. y Jackson, S. E. "The measurement of experienced burnout". Journal of Ocupational Behavior, 12,99-113.

6. Selye, H. (1954). "The general adaptation sindrome and the diseases of adaptation". Journal of Clinical Endocrinology, 6,117-120.

7. $\mathrm{G}$

8. Olembiewski, R.T. (1986). Stress in organizations: Toward a phase model of burnout. New York: Praeger.

9. Golembiewsi, R. T. y Munzenrider, R.F. (1988). Phases of burnout: Developments in concepts and applications. New York: Praeger. 
10. Leiter, M.P. (1988). Burnout as a funtion of communication patterns: a study of a muldidisciplinary mental health team. Group and Organization Stuies, 14(1), 14-22.

11. Cherniss, C. (1980). Profesional burnout in human service organizations. Nueva York: Praeger.

12. Calvete, E. y Villa, A. (1999). "Estrés y Burnout docente: Influencias de variables cognitivas". Revista de Educación, 319,291-303.

13. Fernández Arata, M. (2002). Realidad psicosocial del maestro de primaria. Universidad de Lima, Fondo de Desarrollo Editorial.

14. Calvete, E. y Villa, A. (1997). Proyecto Deusto 14-16: Evaluación e intervención del estrés docente. Bilbao: Mensajero.

15. Kyriacou, C. (1989). The nature and sourcs of stress facing teachers. Comunicación presentada en la Tercera Conferencia Europea de Investigaci6n sobre Aprendizaje e Instrucción. Madrid.

BAREMO POR ENEATIPOS Y PERCENTIBLES DEL MBI - "BURNOUT" EN DOCENTES UNIVERSITARIOS

\begin{tabular}{|c|c|c|c|c|}
\hline $\begin{array}{c}\text { Factores "Burnout" } \\
\text { Eneatipos }\end{array}$ & CE & DP & RP & $\begin{array}{c}\text { Factores "Burnout" } \\
\text { Percentiles }\end{array}$ \\
\hline 9 & $336+$ & $156+$ & $526+$ & $96-100$ \\
\hline 8 & $28-32$ & $13-14$ & $47-51$ & $89-95$ \\
\hline 7 & $23-27$ & $10-12$ & $43-46$ & $77-88$ \\
\hline 6 & $18-22$ & $8-9$ & $38-42$ & $60-76$ \\
\hline 5 & $12-17$ & $5-7$ & $34-47$ & $40-59$ \\
\hline 4 & $7-11$ & $2-4$ & $29-33$ & $23-29$ \\
\hline 3 & $2-6$ & $0-1$ & $25-28$ & $11-22$ \\
\hline 2 & $0-1$ & $0-0$ & $20-24$ & $4-10$ \\
\hline 1 & $0-0$ & $0-0$ & $0-19$ & $0-3$ \\
\hline & $\mathrm{N}=274$ & $\mathrm{~N}=274$ & $\mathrm{~N}=274$ & \\
\hline & $\mathrm{M}=15.02$ & $\mathrm{M}=6.23$ & $\mathrm{M}=35.99$ & \\
\hline & $\mathrm{D} . \mathrm{S}=10.42$ & $\mathrm{D} . \mathrm{S}=5.32$ & $\mathrm{D} . \mathrm{S}=9.07$ & \\
\hline & & & & \\
\hline
\end{tabular}

C. Ponce, 2001. 